\title{
Deep Priors for Ptycho-tomography
}

\author{
Selin Aslan, Zhengchun Liu, Viktor Nikitin, Tekin Bicer, Sven Leyffer and Doga Gursoy
}

Argonne National Laboratory, Lemont, Illinois, United States

Ptychography is a coherent diffraction imaging technique that avoids the limitations introduced by conventional lens-based microscopy. Hence, it has been widely used in many applications in the last decade and is becoming the standard method for sub-20nm x-ray imaging. In ptychography, the object is scanned to collect a series of diffraction patterns from partially overlapping regions. Although, the method has been popularized for 2D imaging, the extension of ptychography into 3D imaging has received a great deal of interest in recent years. The conventional way to recover the refractive properties of the 3D object is to solve the ptychography problem first for each view of the object which is then followed by a 3D tomographic reconstruction. The drawback of this sequential approach is that current ptychographic phase retrieval algorithms require $70 \%$ or more overlap between illuminations. In [Gürsoy, 2017], it has been shown that these constraints can be relaxed by using the intrinsic coupling of information among projections and solving the ptycho-tomography problem jointly. Later in [Aslan, 2019], the alternating direction method of multipliers (ADMM) is used as a generic reconstruction framework to efficiently solve the joint ptycho-tomography problem. ADMM allows to split the problem into parts where each subproblem can be solved with an optimizer. Due to its modular structure, a priori knowledge of the image can also be plugged into the framework where the problem can be split into three parts: ptychography, tomography problem and the regularizer. In [Nikitin, 2019], we used a sparse prior defined in terms of the total variation $(\mathrm{TV})$.

Choosing an appropriate prior for the model has been a topic of many research papers in image processing. While model-based methods solve the optimization problem with an explicit regularization, such as TV, the learning-based methods aim to learn a prior that maps noisy reconstructions to a desirable image. Due to its great success in improving the quality of low-dose images, we incorporate deep priors into the generic reconstruction framework for the joint ptycho-tomography problem where we split the ptychography, tomography problem and the denoiser into three subproblems. We show the effectiveness of our approach via simulated studies.

\section{References}

1. [Gürsoy, 2017]. D. Gürsoy. Direct coupling of tomography and ptychography, Opt. Lett. 42, 31693172 (2017).

2. [Aslan, 2019]. S. Aslan, V. Nikitin, D. J. Ching, T. Bicer, S. Leyffer, and D. Gürsoy, Joint ptychotomography reconstruction through alternating direction method of multipliers, Opt. Express 27, 91289143 (2019).

3. [Nikitin, 2019]. V. Nikitin, S. Aslan, Y. Yao, T. Bicer, S. Leyffer, R. Mokso, and D. Gürsoy. Photonlimited ptychography of 3D objects via Bayesian reconstruction. OSA Continuum 2, 2948-2968, (2019). 